\title{
Modelagem da decomposição aeróbia de lodo de esgoto em solos com diferentes texturas
}

\author{
Cláudia R. Teles ${ }^{1}$, Celso J. Munaro ${ }^{2} \&$ Sérvio T. A. Cassini ${ }^{3}$
}

\begin{abstract}
RESUMO
Buscou-se, neste trabalho, avaliar o efeito do teor de argila sobre o processo de decomposição de lodo de esgoto disposto em solo e propor modelos matemáticos inserindo este efeito em seus parâmetros. Solos com texturas contrastantes originados de uma mesma matriz permitiram estabelecer melhores relações entre o teor de argila, biomassa microbiana e carbono orgânico total e a produção de $\mathrm{CO}_{2}$ do que experimentos similares da literatura em que foram utilizados solos de diferentes matrizes. O efeito do teor de argila se manifestou apenas no período inicial de 7 dias $\left(R^{2}=0,59^{* *}\right)$. Modelos cinéticos foram propostos para modelar a decomposição de lodo em solos com diferentes texturas. Determinaram-se modelos de primeira ordem bifásicos, assumindo que a fase lenta e a rápida não ocorrem simultaneamente, enquanto os parâmetros do modelo correspondentes às fases lenta e rápida foram correlacionados ao teor de argila resultando em modelos matemáticos que evidenciaram o efeito deste fator no processo de decomposição ao mesmo tempo em que permitem prever seu efeito na produção de $\mathrm{CO}_{2}$.
\end{abstract}

Palavras-chave: lodo anaeróbio, teor de argila, respirometria do solo, atividade microbiana, carbono orgânico

\section{Modeling of aerobic decomposition of sewage sludge in soils of different textures}

\begin{abstract}
The objective of this study was to evaluate the effect of clay content on sewage sludge decomposition in soil and to propose mathematical models with clay dependent parameters. Soils with contrasting textures from the same sample matrix allowed better relationships to be derived between clay content, microbial biomass, organic carbon and $\mathrm{CO}_{2}$ production then those usually obtained in the literature. Clay effect was noticed only in the first week $\left(\mathrm{R}^{2}=0.59^{* *}\right)$. Kinetic models were proposed to model sludge decomposition in soils with different textures. Double phase first order models with non-simultaneous time scales were fitted to the data. Model parameters were correlated to clay content, emphasizing the clay effect on sewage sludge decomposition and providing a model for prediction of $\mathrm{CO}_{2}$ production.
\end{abstract}

Key words: anaerobic sludge, clay content, soil respirometry, microbial activity, organic carbon 


\section{INTRODUÇÃO}

A aplicação do lodo de esgoto na agricultura tem sido excelente alternativa de disposição final desse resíduo, principalmente pelo seu alto teor de matéria orgânica e nutrientes, que melhoram as propriedades físicas, químicas e biológicas do solo (Andreoli \& Fernandes, 1999). Os solos sujeitos a aplicação sucessiva de resíduos orgânicos podem acumular carbono em formas orgânicas exercendo assim, importante papel na dinâmica de reações químicas que regulam a fertilidade do solo nos estoques de C e N. Pedroza et al. (2006) constataram que a aplicação de doses de biossólidos exerce efeito significativo na qualidade tecnológica da pluma do algodoeiro. Conhecer a forma como a decomposição de biossólidos ocorre em diferentes solos contribui para definir melhor os procedimentos para sua disposição final.

Segundo a norma NBR 14283 da ABNT (ABNT, 1999) a decomposição dos resíduos orgânicos no solo pode ser determinada de forma indireta, através da medição do $\mathrm{CO}_{2}$ produzido pelos microrganismos, a fim de obter energia para o seu crescimento. A dinâmica do carbono no solo e a sua liberação como $\mathrm{CO}_{2}$ na atmosfera, estão intimamente relacionadas à atividade microbiana, ao suprimento de substratos orgânicos, aos microrganismos e às suas interações com a matriz do solo. Com o exposto, os microrganismos são considerados a força direcionadora ou catalisadora do processo de decomposição. A biomassa microbiana (BMS) é descrita, comumente, como compartimento ativo ou vivo em modelos que simulam a transformação do C orgânico em solos. O tamanho deste compartimento e de outros índices de disponibilidade de substrato afeta diretamente os modelos que descrevem a transformação da matéria orgânica no solo (Wang et al., 2003; Liu et al., 2006). Em Franzluebbers (1999) concluiu-se que não há evidências de que a BMS seja afetada por procedimentos experimentais de secagem e peneiramento do solo, comuns em experimentos de análise de mineralização do carbono.

Existem, na literatura, resultados que confirmam que o teor de argila está altamente relacionado com a concentração de COT no solo. Muller \& Hopper (2004) avaliaram as relações existentes entre o teor de argila e as transformações da matéria orgânica no solo e obtiveram correlação significativa entre o compartimento de carbono húmico e o teor de argila do solo, após um período de incubação. Wang et al. (2003) observaram que durante os estágios iniciais de incubação dos solos, após o reumedecimento dos solos secados ao ar, o C orgânico lábil era mineralizado, não sendo influenciado pela argila; entretanto, nos períodos mais avançados da incubação o conteúdo de argila do solo era o responsável pela redução na taxa de mineralização do $\mathrm{C}$ orgânico. Thomsen et al. (2003), avaliando a produção de $\mathrm{CO}_{2}$ em solos sem adição de matéria orgânica, notaram que as produções de $\mathrm{CO}_{2}$ foram maiores em solos argilosos que em arenosos, porém observaram que as taxas constantes de mineralização de carbono foram maiores em solos arenosos. Uma explicação para este fenômeno é a alta concentração de argila, que pode impedir a mineralização da matéria orgânica do solo, possivelmente através da proteção física da decomposição da adsorção superficial (Oades, 1988). Neste contexto, o uso de solos originados de uma mesma matriz e com teores de argila contrastantes, permitiu avaliar o efeito do teor de argila sobre as taxas de produção de $\mathrm{CO}_{2}$. A metodologia empregada neste artigo ensejou avaliar, de forma mais precisa este efeito, que em experimentos similares da literatura, utilizando-se solos de diferentes matrizes.

Modelos matemáticos dados por uma equação de primeira ordem, são os mais usados para descrever a dinâmica da decomposição do substrato ou evolução de $\mathrm{CO}_{2}$. Uma suposição implícita da cinética de primeira ordem é que a taxa de mineralização do $\mathrm{C}$ depende da disponibilidade do substrato (Whitmore, 1996). Alguns modelos sobre a decomposição da matéria orgânica nos solos incorporam este efeito diferenciado da argila sobre a mineralização do C orgânico do solo (Muller \& Hopper, 2004; Thomsen et al., 2003). Nos modelos aqui propostos a correlação entre os parâmetros dos modelos e o teor de argila para as fases rápida e lenta do processo de decomposição, foi computada, permitindo a obtenção de modelos de regressão relacionando o teor de argila com os parâmetros por ela influenciados.

\section{MATERIAL E MÉTODOS}

\section{Características e preparação do solo}

A partir de um único tipo de solo, de textura predominante argilosa, coletado a uma profundidade de 0 a $20 \mathrm{~cm}$ da superfície do solo, de área sem cultivo, localizada no município de Vitória, no Estado do Espírito Santo, foram construídas três classes texturais altamente contrastantes. Às amostras de solo de fração argilosa foi acrescentado um percentual de areia, em proporções adequadas para a obtenção das classes texturais desejadas, segundo o triângulo de classificação empregado pelo Departamento de Agricultura Americano (USDA), adotado pela Sociedade Brasileira de Ciência do Solo, totalizando quatro classes texturais originadas da mesma matriz. A areia, utilizada para compor as amostras restantes foi obtida do solo original através da lavagem em peneira $n^{\circ} 200$ da ASTM (0,075 mm) e separação da fração argila, que retém somente a areia e deixa passar a argila e o silte (NBR 7181/1984). As classes texturais compostas a partir de amostras de $100 \mathrm{~g}$ de solo original foram:

1. Solo argiloso (original), composto de $71 \%$ de argila + silte e $29 \%$ de areia;

2. Solo argilo-arenoso, composto de 49,9\% de argila + silte e 50,1 \% de areia;

3. Solo franco-arenoso, composto de $30 \%$ de argila + silte e $70 \%$ de areia;

4. Solo arenoso, composto de $10 \%$ de argila + silte e $90 \%$ de areia.

A umidade das amostras de solo foi corrigida a aproximadamente $40-60 \%$ da capacidade de campo, utilizando-se extrato de solo; as amostras de solo foram coletadas de uma profundidade de $0-20 \mathrm{~cm}$, secadas ao ar e peneiradas em peneira de malha $2 \mathrm{~mm}$ (ABNT). As análises químicas e granulométrica de rotina foram realizadas de acordo com métodos empregados pela EMBRAPA (1997), conforme apresentado na Tabela 1, adaptada de Teles et al. (2007). 
Tabela 1. Características químicas e granulometria do solo original

\begin{tabular}{|c|c|c|c|c|c|c|c|c|c|c|c|}
\hline \multirow{2}{*}{$\mathrm{pH} \mathrm{CaCl}{ }_{2}$} & \multirow{2}{*}{$\begin{array}{c}\text { MO } \\
\text { dag } \mathrm{kg}^{-1}\end{array}$} & $\mathrm{H}+\mathrm{Al}$ & $\mathrm{Al}^{3+}$ & SB & CTC & V & $\mathbf{m}$ & Areia grossa & Areia fina & Silte & Argila \\
\hline & & \multicolumn{4}{|c|}{$\mathrm{cmol}_{\mathrm{c}} \mathrm{dm}^{-3}$} & \multicolumn{6}{|c|}{ dag $\mathrm{kg}^{-1}$} \\
\hline 5,6 & 0,14 & 1,86 & 0,01 & 3,51 & 5,37 & 65,4 & 0,28 & 19 & 10 & 21 & 50 \\
\hline
\end{tabular}

\section{Características e preparação do lodo}

O lodo de esgoto, fonte de matéria orgânica utilizada no experimento, proveio de um reator anaeróbio do tipo UASB. A caracterização física e química do lodo de esgoto foi realizada de acordo com APHA (1995). Os resultados expressos em base seca estão descritos na Tabela 2, adaptada de Teles et al. (2007). Inicialmente, o lodo foi desaguado em leito de secagem durante $48 \mathrm{~h}$, secado a $40{ }^{\circ} \mathrm{C}$, moído, peneirado em peneira de $2 \mathrm{~mm}$ (ABNT) e só então armazenado em frasco de vidro.

Tabela 2. Caracterização física e química do lodo de esgoto anaeróbio

\begin{tabular}{|c|c|c|c|c|c|c|}
\hline \multirow{2}{*}{$\mathrm{pH} \mathrm{H} \mathrm{H}_{2} \mathrm{O}$} & $\begin{array}{l}\text { Umidade } \\
\left(65^{\circ} \mathrm{C}\right)\end{array}$ & C total & $\mathrm{N}$ total & $\mathrm{Ca}^{2+}$ & $\mathbf{P}$ & \multirow{2}{*}{$\begin{array}{c}\mathrm{K}^{+} \\
\mathrm{mg} \mathrm{kg}^{-1}\end{array}$} \\
\hline & $\%$ & \multicolumn{4}{|c|}{$\mathrm{g} \mathrm{kg}^{-1}$} & \\
\hline 5,7 & 9,4 & 307,3 & 33,7 & 127 & 4,4 & 783 \\
\hline
\end{tabular}

\section{Ensaio de incubação}

Quatro amostras (uma para cada tipo de solo) foram preparadas para uma medida única da BMS e COT e sacrificadas após sete dias de incubação. Essas amostras, de $1000 \mathrm{~g}$ de solo seco, foram pesadas dentro de potes de vidro e ajustadas a 40-60\% CC pela adição de água deionizada e extrato de solo. Os potes foram incubados durante sete dias.

De forma simultânea, amostras de $100 \mathrm{~g}$ de solo umedecido com um volume de água destilada adequado para atingir 40-60\% da capacidade de campo, foram dispostas em potes de vidro herméticos de $1 \mathrm{~L}$; posteriormente, misturou-se o lodo de esgoto ao solo, na dose correspondente de 1,2 g base seca por frasco contendo $100 \mathrm{~g}$ de solo, definida para este estudo.

A incubação foi conduzida em temperatura média de $28^{\circ} \mathrm{C}$, no escuro, durante 108 dias. Na Tabela 3 se encontram as medidas da BMS e COT para o solo após 7 dias de incubação, antes da adição de lodo, e a produção média diária de $\mathrm{CO}_{2}$ na primeira semana de incubação, após a adição de lodo. Realizou-se uma medição por repetição para COT e apenas uma por tratamento para a BMS.

Tabela 3. Medidas de biomassa microbiana (BMS), carbono orgânico total (COT) e produção média diária de $\mathrm{CO}_{2}{ }^{*}$

\begin{tabular}{|c|c|c|c|}
\hline Tratamento & $\begin{array}{c}\mathrm{CO}_{2} \\
\mathrm{mg} \mathrm{CO} \mathrm{CO}_{2} 100 \mathrm{~g}^{-1}\end{array}$ & $\begin{array}{c}\text { COT } \\
\text { g C kg-1 }\end{array}$ & $\begin{array}{c}\text { BMS } \\
\mathrm{mg} \mathrm{C} \mathrm{kg}^{-1}\end{array}$ \\
\hline Solo argiloso (1) & $16,5 \pm 1,82$ & $7,3 \pm 0,3$ & 258,08 \\
\hline Solo argilo-arenoso (2) & $17,6 \pm 1,43$ & $5,0 \pm 0,9$ & 225,52 \\
\hline Solo franco-arenoso (3) & $21,9 \pm 1,75$ & $4,1 \pm 0,1$ & 210,54 \\
\hline Solo arenoso (4) & $21,9 \pm 1,75$ & $1,6 \pm 0,2$ & 181,92 \\
\hline
\end{tabular}

* Medidas da BMS e COT para o solo após 7 dias de incubação, antes da adição do lodo, e produção média diária de $\mathrm{CO}_{2}$ na primeira semana de incubação, após a adição do lodo.

\section{Medição do $\mathrm{CO}_{2}$ produzido}

A produção de $\mathrm{CO}_{2}$ foi determinada de forma indireta, por meio da quantificação do $\mathrm{CO}_{2}$ produzido pelos microrganis- mos decompositores da matéria orgânica adicionada sob a forma de lodo de esgoto (ABNT, 1999; CETESB, 1990). Para cada pote de vidro utilizou-se um pequeno frasco de polietileno de $50 \mathrm{~mL}$ de capacidade contendo $20 \mathrm{~mL}$ de solução $\mathrm{NaOH}$ de concentração conhecida (aproximadamente 0,25 mol L-1), para a absorção do $\mathrm{CO}_{2}$ produzido pelos microrganismos do solo. A solução de $\mathrm{NaOH}$ contida no interior dos frascos de plástico era trocada periodicamente por uma nova solução de $\mathrm{NaOH}$, isenta de íons carbonato, para a captura de $\mathrm{CO}_{2}$ durante um período preestabelecido; posterior à adição de lodo, as medidas produzidas pelo solo e pelo lodo foram feitas diariamente, nos primeiros seis dias e com maior intervalo após este período, durante 108 dias de incubação. $\mathrm{O} \mathrm{CO}_{2}$ produzido foi quantificado pelo método condutimétrico (Rodella \& Saboya, 1999). Realizou-se a calibração para a determinação do $\mathrm{CO}_{2}$ através da utilização de soluções de $\mathrm{NaOH}$ e $\mathrm{Na}_{2} \mathrm{CO}_{3}$, de concentração conhecida, considerando-se que a solução de $\mathrm{NaOH}$ parcialmente neutralizada pelo $\mathrm{CO}_{2}$, pode ser comparada com à mistura de soluções padrão de $\mathrm{NaOH}$ e $\mathrm{Na}_{2} \mathrm{CO}_{3}$.

\section{Análises químicas}

Determinou-se a matéria orgânica total do lodo através da perda de massa após incineração em mufla a $550{ }^{\circ} \mathrm{C}$, durante 1 h; o nitrogênio total foi definido pelo método Kjeldahl; o pH em água o foi pelo método potenciométrico; os elementos $\mathrm{Ca}, \mathrm{P}$ e $\mathrm{K}$ foram mensurados segundo o método US-EPA 3051, com determinação por espectrometria de emissão atômica (ICp-AES) e fotometria de chama. As análises químicas e granulométricas do solo foram realizadas de acordo com os métodos empregados pela EMBRAPA (1997). O carbono orgânico do solo foi determinado segundo o método Walkley \& Black, descrito por Nelson \& Sommers (1982) e a Biomassa Microbiana (BMS), pelo método de fumigação e extração (Vance et al., 1987).

\section{Análise dos resultados}

Obtiveram-se os dados experimentais de produção de $\mathrm{CO}_{2}$ do lodo por meio da subtração da quantidade de $\mathrm{CO}_{2}$ produzido nas amostras contendo lodo de esgoto, das quantidades produzidas nos frascos de controle. O resultado dessa subtração foi atribuído à mineralização do C-orgânico do lodo de esgoto; já os dados experimentais de produção de $\mathrm{CO}_{2}$ do controle foram obtidos subtraindo-se a quantidade de $\mathrm{CO}_{2}$ produzido nas amostras do controle contendo solo das quantidades médias produzidas nos frascos do branco. Apesar da possibilidade de ocorrência devido à decomposição da matéria orgânica do solo, o efeito "priming” foi desprezado no presente estudo. O uso do software Matlab ${ }^{\circledR}$, versão 6.5 (Math Works, US) obtém os parâmetros cinéticos dos modelos matemáticos de primeira e segunda ordens, utilizando-se o valor da produção de $\mathrm{CO}_{2}$ que melhor se ajusta a cada experimento, fazendo cálculos de erros e gerando gráficos que 
permitem visualizar mais facilmente as informações necessárias para as análises. A função que obtém os parâmetros executa um programa de otimização não linear que minimiza o erro padrão entre os valores medidos e estimados pelo modelo. O cálculo dos parâmetros ótimos de aproximação de primeira e segunda ordens, foi realizado a partir da função "fminsearch" do Matlab. O experimento se compôs de 4 tratamentos, 3 repetições com adição de lodo, 3 repetições sem adição de lodo (controle) e 3 brancos (somente $\mathrm{NaOH}$ ), totalizando 27 unidades experimentais. O software Matlab ${ }^{\circledR}$ também foi empregado para se realizar as análises de variância, de regressão e o teste $\mathrm{F}(\alpha=0,05)$.

\section{RESULTADOS E DISCUSSÃO}

\section{Produção acumulada de $\mathrm{CO}_{2}$}

Utilizou-se a produção de $\mathrm{CO}_{2}$ do lodo de esgoto adicionado ao solo e incubado durante 108 dias, para as análises e discussões a seguir. Na Figura 1 se acham as produções acumuladas de $\mathrm{CO}_{2}$ do lodo de esgoto, em diferentes tipos de solo; nos solos franco-arenosos e arenosos, as produções foram de 248,8 e $235 \mathrm{mg}$ de $\mathrm{CO}_{2}$, respectivamente; já nos solos argilo-arenosos e argilosos as produções foram de 215 e 207,6 mg de $\mathrm{CO}_{2}$, respectivamente. A Tabela 4 apresenta os resultados da análise de variância da produção acumulada de $\mathrm{CO}_{2}$. Na coluna teste de média, o número 1 significa que houve diferença significativa entre os tratamentos da coluna tratamentos. Os tratamentos 1, 2, 3 e 4 correspondem aos solos argiloso, argilo-arenoso, franco-arenoso e arenoso, respectivamente. O símbolo "\&" representa todos os tratamentos. Houve diferença significativa entre tratamentos do dia 1 ao dia 9, menos nos dias 3 e 4; tal diferença não foi significativa entre os tratamentos 1 e 2 (argilosos) nem entre os tratamentos 3 e 4 (arenosos); do dia 11 ao 36 dia, não ocorreu diferença significativa entre os tratamentos, porém do quadragésimo segundo dia ao centésimo oitavo dia se constataram tratamentos com médias com diferença significativa. As produções médias diárias de $\mathrm{CO}_{2}$ durante os 7 primeiros dias de incubação variaram de 16,5 a $21,9 \mathrm{mg} \mathrm{CO}_{2} 100 \mathrm{~g}^{-1}$ solo dia-1 para os quatro solos (Tabela 3). Tem-se, na Figura 2, a análise das médias dessas produções observando-se, novamente, que os tratamentos 1 e 2 e os tratamentos 3 e 4 não diferiram estatisticamente entre si; na semana seguinte não houve diferença estatística entre os tratamentos; tais resultados de análise de variância indicam o período no qual a correlação entre o teor de argila e a produção de $\mathrm{CO}_{2}$ pode ser estabelecida e computada, deixando claro que o efeito do teor de argila se manifesta apenas no período inicial.

\section{Análises de regressão}

Considerando que solos com diferentes texturas apresentaram comportamento diferenciado em relação à produção de $\mathrm{CO}_{2}$ no período de 1 a 7 dias, avaliaram-se as relações entre o teor de argila, produção diária média de $\mathrm{CO}_{2}$ dos primeiros 7 dias, após a adição de lodo COT e BMS do solo, medidos imediatamente antes da adição do lodo, e suas combi-

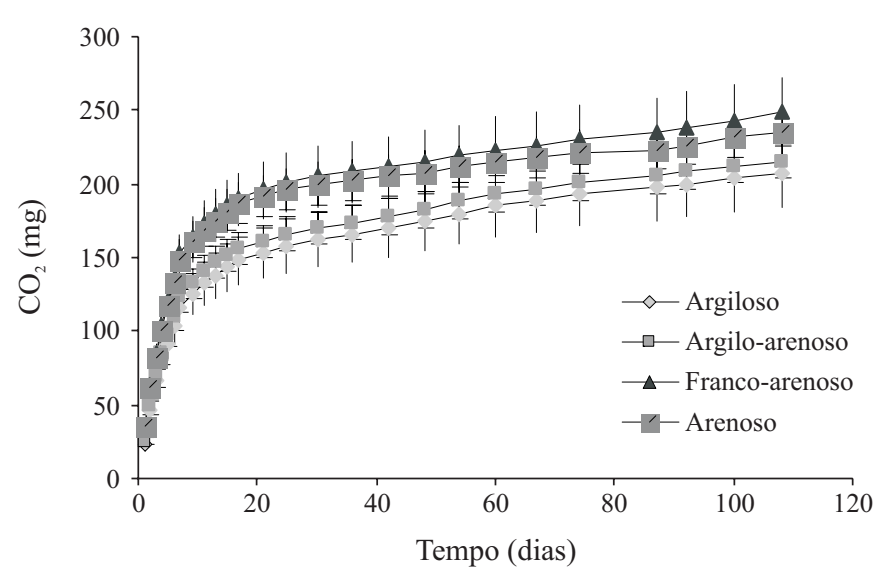

Figura 1. Produção acumulada de $\mathrm{CO}_{2}$

Tabela 4. Resultado da ANOVA para a produção acumulada

\begin{tabular}{|c|c|c|c|c|c|c|c|}
\hline \multirow{2}{*}{$\begin{array}{c}\text { Dias } \\
1,5,6,7,9\end{array}$} & \multicolumn{2}{|c|}{ Tratamentos* } & \multirow{2}{*}{$\begin{array}{c}\text { Teste de } \\
\text { média } \\
-1\end{array}$} & \multirow{2}{*}{$\begin{array}{c}\text { Dias } \\
48,54,60,67\end{array}$} & \multicolumn{2}{|c|}{ Tratamentos } & \multirow{2}{*}{$\begin{array}{c}\text { Teste de } \\
\text { média } \\
-1\end{array}$} \\
\hline & 1 & 2 & & & 1 & 2 & \\
\hline $1,5,6,7,9$ & 1 & 3 & 1 & $48,54,60,67$ & 1 & 3 & 1 \\
\hline $1,5,6,7,9$ & 1 & 4 & 1 & $48,54,60,67$ & 1 & 4 & 1 \\
\hline $1,5,6,7,9$ & 2 & 3 & 1 & $48,54,60,67$ & 2 & 3 & 1 \\
\hline $1,5,6,7,9$ & 2 & 4 & 1 & $48,54,60,67$ & 2 & 4 & 1 \\
\hline $1,5,6,7,9$ & 3 & 4 & -1 & $48,54,60,67$ & 3 & 4 & -1 \\
\hline 2 & 1 & 2 & -1 & 74 & \& & \& & -1 \\
\hline 2 & 1 & 3 & 1 & 87,92 & 1 & 2 & -1 \\
\hline 2 & 1 & 4 & -1 & 87,92 & 1 & 3 & -1 \\
\hline 2 & 2 & 3 & -1 & 87,92 & 1 & 4 & 1 \\
\hline 2 & 2 & 4 & -1 & 87,92 & 2 & 3 & -1 \\
\hline 2 & 3 & 4 & -1 & 87,92 & 2 & 4 & 1 \\
\hline 3 & $\&$ & $\&$ & -1 & 87,92 & 3 & 4 & 1 \\
\hline 4 & \& & $\&$ & -1 & 100 & 1 & 2 & -1 \\
\hline 11 & $\&$ & $\&$ & -1 & 100 & 1 & 4 & -1 \\
\hline 13 & $\&$ & \& & -1 & 100 & 2 & 3 & 1 \\
\hline 15 & \& & $\&$ & -1 & 100 & 2 & 4 & -1 \\
\hline 17 & $\&$ & $\&$ & -1 & 100 & 3 & 4 & -1 \\
\hline 21 & $\&$ & $\&$ & -1 & 108 & 1 & 2 & -1 \\
\hline 25 & \& & $\&$ & -1 & 108 & 1 & 3 & 1 \\
\hline 30 & $\&$ & $\&$ & -1 & 108 & 1 & 4 & -1 \\
\hline 36 & $\&$ & $\&$ & -1 & 108 & 2 & 3 & 1 \\
\hline 42 & 1 & 2 & -1 & 108 & 2 & 4 & -1 \\
\hline 42 & 1 & 3 & -1 & 108 & 3 & 4 & -1 \\
\hline 42 & 1 & 4 & 1 & & & & \\
\hline 42 & 2 & 3 & -1 & & & & \\
\hline 42 & 2 & 4 & 1 & & & & \\
\hline 42 & 3 & 4 & -1 & & & & \\
\hline
\end{tabular}

* Os tratamentos 1, 2, 3 e 4 referem-se aos solos argiloso, argilo-arenoso, franco-arenoso e arenoso, respectivamente

nações. A Tabela 5 resume essas análises observando-se, de início, forte relação entre a COT e BMS e o teor de argila, que é um resultado esperado. Notou-se também forte associação negativa entre a produção diária de $\mathrm{CO}_{2}$ da primeira semana e o teor de argila $\left(\mathrm{R}^{2}=0,59 * *\right)$; a relação entre $\mathrm{CO}_{2} /$ COT e o teor de argila foi negativa e alta $\left(\mathrm{R}^{2}=0,92^{* *}\right)$, e indica que os solos mais argilosos produziram menor quantidade de $\mathrm{CO}_{2}$, considerando o COT do respectivo solo; a relação entre $\mathrm{CO}_{2} / \mathrm{BMS}$ com o teor de argila é negativa $\left(\mathrm{R}^{2}=0,88^{* *}\right)$. 


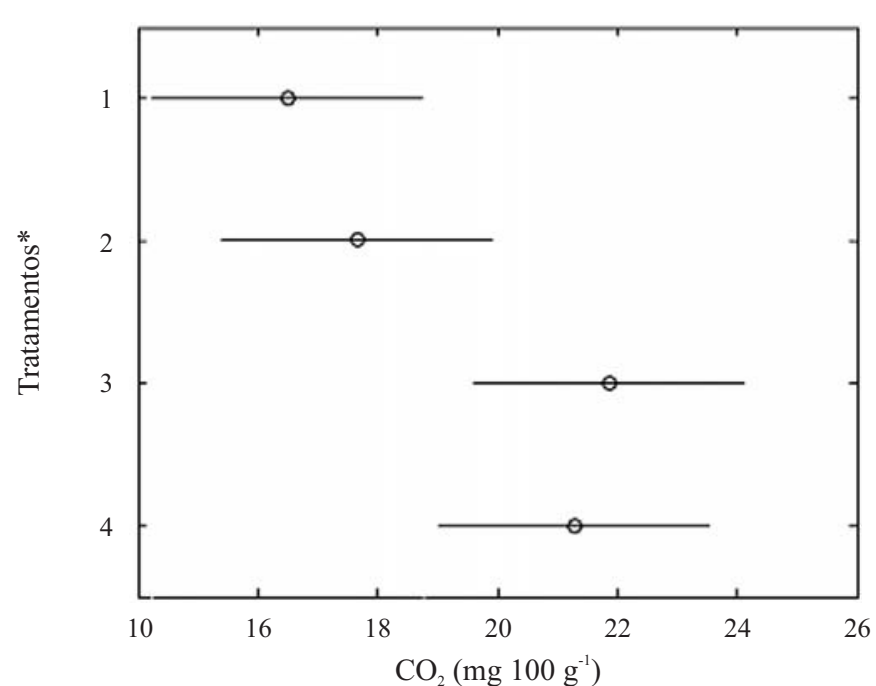

* Os tratamentos 1, 2, 3 e 4 referem-se aos solos argiloso, argilo-arenoso, franco-arenoso e arenoso, respectivamente

Figura 2. Comparação das produções médias diárias de $\mathrm{CO}_{2}$ do período de 1 a 7 dias

Tabela 5. Testes de regressão entre os fatores produção diária média de $\mathrm{CO}_{2}$, $\mathrm{COT}$ e BMS e o teor de argila

\begin{tabular}{ccc}
\hline Fator $^{1}$ & Modelos ajustados & $\mathbf{R}^{2}$ \\
$\mathrm{CO}_{2}$ & $\mathrm{Y}=23,04-0,09 \mathrm{X}$ & $0,59 *$ \\
$\mathrm{COT}$ & $\mathrm{Y}=0,90+0,09 \mathrm{X}$ & $0,94 * *$ \\
$\mathrm{CO}_{2} / \mathrm{COT}$ & $\mathrm{Y}=4,24-0,04 \mathrm{X}$ & $0,92 * *$ \\
$\mathrm{BMS}$ & $\mathrm{Y}=170+1,18 \mathrm{X}$ & $0,96 * *$ \\
$\mathrm{CO}_{2} / \mathrm{BMS}$ & $\mathrm{Y}=0,13-0,001 \mathrm{X}$ & $0,88^{* *}$ \\
\hline
\end{tabular}

${ }_{1}^{1}$ COT - Carbono orgânico total; RMS - Biomassa microbiana

${ }^{*} e$ ** indicam significância $p<0,05$ e $p<0,01$, respectivamente

Os resultados deixam claro que, apesar de possuírem maior COT e BMS, os solos com maior teor de argila produziram menor quantidade de $\mathrm{CO}_{2}$ no período considerado. Em Khalil et al. (2005), diferentes tipos de matéria orgânica foram adicionados a solos com diferentes caraterísticas, e concluiu-se que a mineralização do carbono era mais afetada pelo tipo de matéria orgânica do que pelos fatores do solo, para os quais foi obtida baixa correlação não significativa. No experimento conduzido neste artigo, o uso de uma mesma matriz para compor os diferentes solos e a aplicação do mesmo tipo e quantidade de lodo nos quatro tratamentos, permitiu isolar o efeito do teor de argila sobre o processo de decomposição.

\section{Modelos cinéticos}

Embora modelos mais sofisticados possam ser utilizados (Whitmore, 1996) para modelar a produção de $\mathrm{CO}_{2}$ de processos de decomposição de lodo, preferiram-se neste trabalho modelos de primeira ordem de uma ou mais fases, os quais permitem explorar, de forma mais simplificada, o efeito do teor de argila em seus parâmetros. O modelo utilizado foi

$$
\mathrm{CO}_{2}(\mathrm{t})= \begin{cases}\mathrm{C}_{1}\left(1-\mathrm{e}^{-\mathrm{k}_{1} t}\right) & 0 \leq \mathrm{t}<\tau \\ \mathrm{C}_{1}\left(1-\mathrm{e}^{-\mathrm{k}_{1} t}\right)+\mathrm{C}_{2}\left(1-\mathrm{e}^{-\mathrm{k}_{2}(t-\tau)}\right) & \mathrm{t} \geq \tau\end{cases}
$$

Este modelo tem duas fases associadas a $\mathrm{k}_{1}$ e $\mathrm{k}_{2}$, respectivamente, que não ocorrem de forma simultânea (Teles et al., 2007). A escolha deste modelo partiu da análise da Fi- gura 3, na qual se observa que um modelo de apenas uma fase não representaria adequadamente a curva dos dados medidos. Análise similar foi realizada por Martines et al. (2006), com a adição de lodo de curtume ao solo, no qual o processo de mineralização do carbono adicionado foi dividido em uma fase rápida de 6 dias e outra fase lenta na sequência. O parâmetro $\tau$ se refere ao atraso de tempo entre as fases rápida e lenta; seu valor foi escolhido de forma a minimizar o erro padrão entre a curva medida e a simulada, tendo sido $\tau=15$ dias o melhor valor obtido. Na Figura 3 se tem o modelo de primeira ordem monofásico que se ajusta aos dados dos primeiros vinte dias do período de incubação, e o modelo de primeira ordem bifásico, que se ajusta aos dados de todo o período de 108 dias.

Os parâmetros dos modelos de primeira ordem da fase rápida foram ajustados a cada uma das repetições de cada tratamento, usando-se os dados dos primeiros 15 dias; lançou-se mão do erro entre os dados medidos e a curva do modelo da fase rápida a fim de ajustar os parâmetros do modelo correspondente à fase lenta. Na Figura 3 são mostrados a simulação e os dados medidos para uma repetição do tratamento com $70 \%$ de teor de argila e, na Tabela 6 os parâmetros deste modelo para todos os tratamentos: quatro

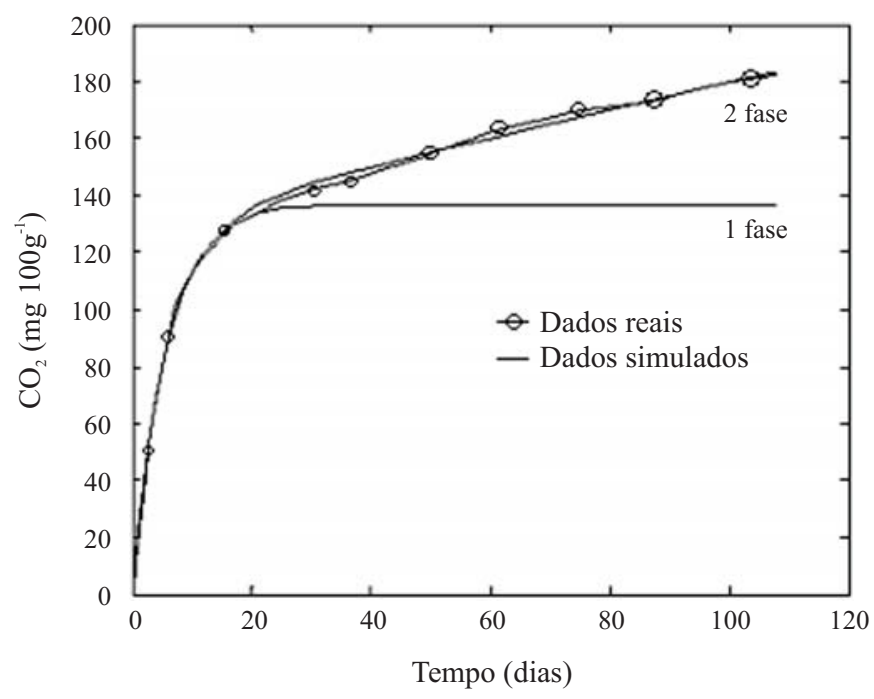

Figura 3. Dados medidos e simulados para o tratamento 1, com de teor de argila de $70 \%$

Tabela 6. Parâmetros do modelo

\begin{tabular}{cccccrc}
\hline Teor de argila & $\mathbf{C}_{1}$ & $\mathbf{k}_{1}$ & $\mathbf{C}_{2}$ & $\mathbf{k}_{2}$ & \multicolumn{1}{c}{ Erro } & $\mathbf{r}$ \\
70 & 136,8 & $-0,175$ & 546,5 & $-0,0010$ & 10,00 & 0,999 \\
70 & 158,9 & $-0,187$ & 334,7 & $-0,0019$ & 9,20 & 1,000 \\
70 & 164,3 & $-0,194$ & 101,0 & $-0,0106$ & 5,85 & 1,000 \\
50 & 152,1 & $-0,176$ & 446,4 & $-0,0013$ & 9,60 & 0,999 \\
50 & 172,5 & $-0,192$ & 148,8 & $-0,0049$ & 9,26 & 1,000 \\
50 & 161,5 & $-0,195$ & 141,3 & $-0,0056$ & 7,71 & 1,000 \\
30 & 216,3 & $-0,189$ & 365,0 & $-0,0018$ & 7,96 & 1,000 \\
30 & 181,8 & $-0,190$ & 1478,5 & $-0,0003$ & 10,56 & 0,999 \\
30 & 197,5 & $-0,196$ & 1091,2 & $-0,0005$ & 9,94 & 1,000 \\
10 & 184,6 & $-0,183$ & 1034,8 & $-0,0004$ & 10,79 & 0,999 \\
10 & 210,1 & $-0,199$ & 380,1 & $-0,0012$ & 9,38 & 1,000 \\
10 & 187,7 & $-0,186$ & 272,5 & $-0,0017$ & 10,55 & 0,999 \\
\hline
\end{tabular}


teores de argila com três repetições cada um. O erro padrão e a correlação são igualmente indicados para cada modelo, todos com $\tau=15$ dias.

Cada conjunto de quatro parâmetros representa um dos tratamentos, com determinado teor de argila. A obtenção de um único modelo cujos parâmetros sejam uma função do teor de argila, é um resultado altamente desejável; a fim de se verificar esta possibilidade calcula-se a correlação de cada um dos quatro parâmetros com os teores de argila, conforme indicado na Tabela 7. Os resultados desta Tabela são coerentes com as análises de variância apresentadas na Tabela 4, nas quais se verificou, após o dia 9, que não havia diferença estatística entre a maior parte dos tratamentos para valores de produção acumulada. Os parâmetros $k_{1}$ e $C_{1}$ estão associados à fase rápida das primeiras duas semanas, em que o primeiro parâmetro, relacionado à taxa, quase não varia; daí, a baixa correlação com o teor de argila; já o parâmetro $\mathrm{C}_{1}$ está ligado à produção de $\mathrm{CO}_{2}$ e seu valor aumenta com a redução do teor de argila, fato coerente com a relação já calculada entre o $\mathrm{CO}_{2}$ e o teor de argila apresentada na Tabela 5. Os parâmetros $k_{2}$ e $\mathrm{C}_{2}$, associados à fase lenta, não têm correlação significativa com o teor de argila, o que também é coerente com a análise de variância da Tabela 4, na qual se verificou que a maior parte dos tratamentos não diferia estatisticamente após a primeira semana de incubação.

Portanto, um modelo cinético que considera o efeito do teor de argila, pode ser proposto para o período inicial. Adotando-se para $\mathrm{k}_{1} \mathrm{o}$ valor médio da Tabela $6, \mathrm{k}_{1}=-0,19$ e para $C_{1}$ o valor dado pelo modelo de regressão, tem-se: $C_{1}(A)=-0,80 A+209$, donde A é o teor de argila; simulações usando-se diferentes valores de A podem ser realizadas. Observa-se a baixa sensibilidade deste parâmetro à variação da argila, dada por

$$
\frac{\mathrm{dC}_{1}(\mathrm{~A})}{\mathrm{dA}}=-0,8
$$

A Figura 4 mostra o resultado da simulação deste modelo para o período de 0 a 17 dias, comparado com os valores médios dos quatro tratamentos. O erro padrão do modelo foi de $12,5,27,2$, 30,7 e 27,5 para os teores de argila de 70, 50, 30 e $10 \%$, respectivamente. Este erro mede o erro quadrático entre a curva obtida com dados reais e a obtida por simulação, permitindo verificar qual o modelo que melhor se ajusta aos dados, no caso, o com teor de argila de $70 \%$. Outras medidas de cálculo do erro podem ser utilizadas, como por exemplo, usando a norma infinita, para obter o erro máximo entre as curvas.

Tabela 7. Correlação dos parâmetros do modelo com o teor de argila

\begin{tabular}{ccc}
\hline Parâmetro & $\mathbf{r}$ & $\mathbf{p}<\mathbf{0 , 0 5}$ \\
$\mathrm{C}_{1}$ & $-0,7792$ & 0,0028 \\
$\mathrm{k}_{1}$ & 0,2629 & 0,4091 \\
$\mathrm{C}_{2}$ & $-0,3833$ & 0,2188 \\
$\mathrm{k}_{2}$ & $-0,5101$ & 0,0902 \\
\hline
\end{tabular}

A.

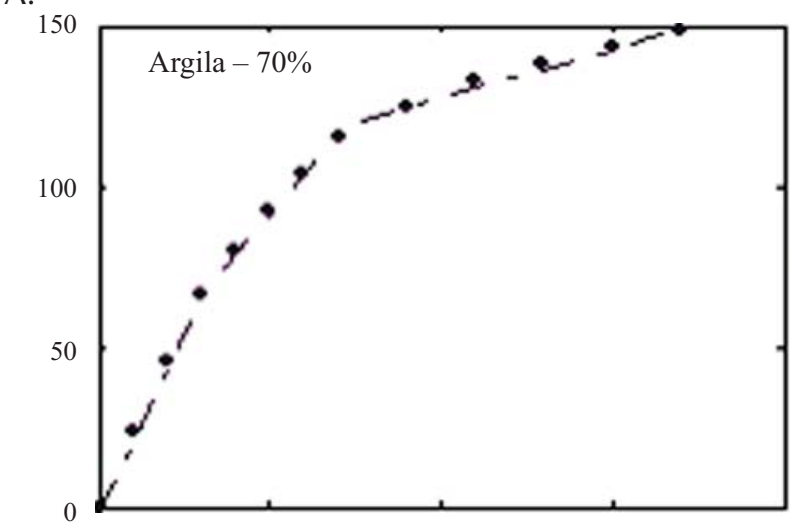

B.

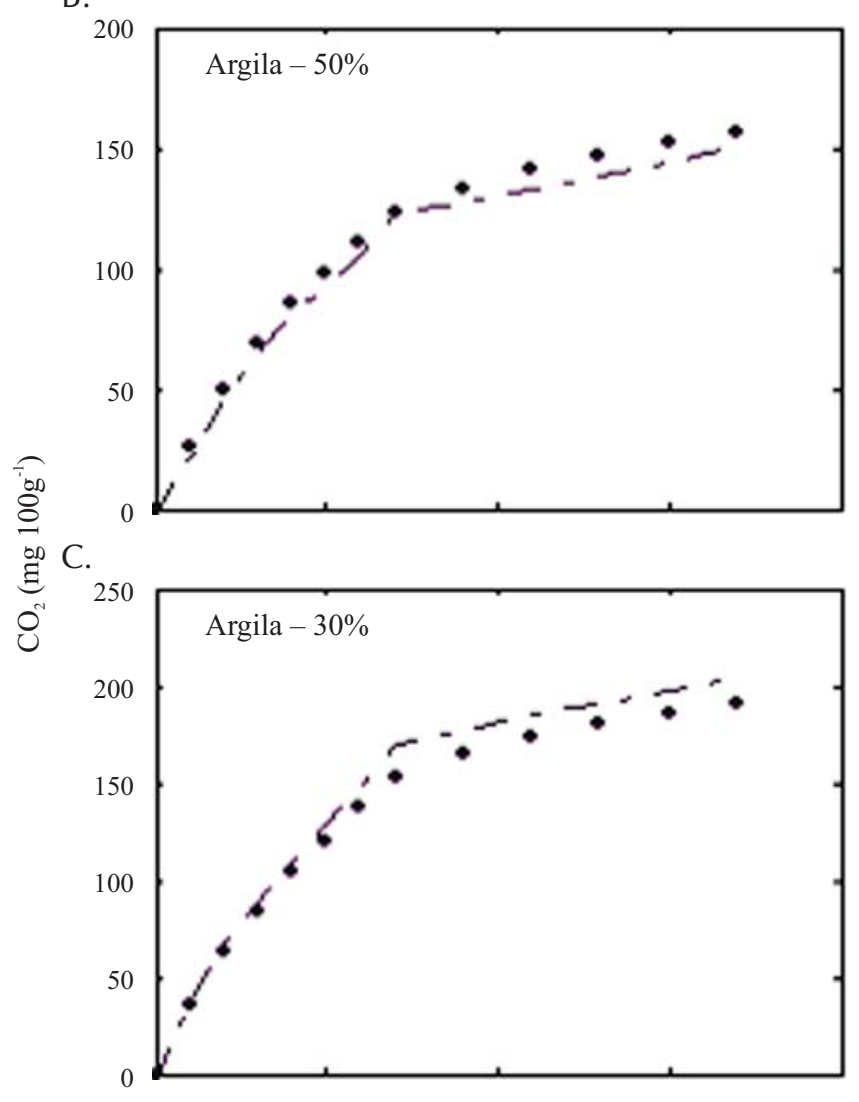

D.

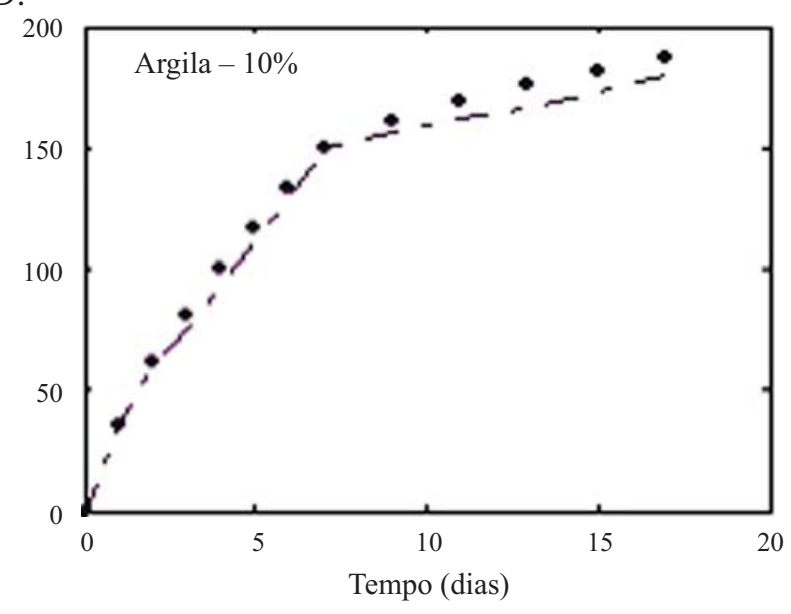

Figura 4. Dados reais médios (•) e simulados (--) para solos com quatro teores de argila 


\section{CONCLUSÕES}

1. A metodologia empregada permitiu a obtenção de altas correlações entre o teor de argila e a produção de $\mathrm{CO}_{2}$ $\left(\mathrm{R}^{2}=0,59 * *\right)$, a BMS $\left(\mathrm{R}^{2}=0,96^{* *}\right)$ e o COT do solo $\left(\mathrm{R}^{2}=0,94^{* *}\right)$.

2. O efeito de fatores do solo sobre o processo de decomposição do lodo de esgoto pode ser melhor quantificado através do uso de uma única matriz de solo para os diferentes tratamentos.

3. O efeito do teor de argila sobre as diferentes fases do processo de decomposição foi incorporado aos parâmetros dos modelos cinéticos, permitindo prever a produção de $\mathrm{CO}_{2}$ para solos com diferentes texturas.

4. O parâmetro do modelo associado ao teor de argila não tem grande sensibilidade, de modo que pequenas variações neste teor pouco afetam a produção de $\mathrm{CO}_{2}$.

\section{AGRADECIMENTOS}

Os autores agradecem a CAPES pela bolsa de doutorado concedida e ao Banco Nordeste pelos recursos alocados neste projeto.

\section{LITERATURA CITADA}

ABNT - Associação Brasileira de Normas Técnicas, NBR 14283. Resíduos em solos - Determinação da biodegradação pelo método respirométrico,1999.

Andreoli, C. V.; Fernandes, F. Reciclagem de biossólidos: transformando problemas em soluções. Curitiba: SANEPAR, FINEP, 1999, 288p.

APHA - American Public Health Association. Standard methods for the examination of water and wastewater. 19.ed. Washington: APHA, AWWA, WPCR, 1995. 1134p.

CETESB - Companhia de Tecnologia e Saneamento Ambiental. L6.350 - Solos - Determinação da biodegradação de resíduos - Método Respirométrico de Bartha, 1990.

EMBRAPA - Empresa Brasileira de Pesquisa Agropecuária. Centro Nacional de Pesquisa de Solos (RJ). Manual de métodos de análise de solos. 2.ed., Rio de Janeiro: EMBRAPA, 1997. 212p.

Franzluebbers, A. J. Potential C and N mineralization and microbial biomass from intact and increasingly disturbed soils of varying texture. Soil Biology and Biochemistry, v.31, n.8, p.1083-1090, 1999.
Khalil, M. I.; Hossain, M. B.; Schmidhalter, U. Carbon and nitrogen mineralization in different upland soils of subtropic treated with organic materials. Soil Biology and Biochemestry, v.37, p.1507-1518, 2005.

Liu, H. S.; Li, L. H.; Hang, G. X.; Huang, J. H.; Sun, J. X.; Wang, H. Y. Respiratory substrate availability plays a crucial role in the response of soil respiration to environmental factor. Applied Soil Ecology, v.32, p.284-292, 2006.

Martines, A. M.; Andrade, C. A.; Cardoso, E. J. B. N. Mineralização do carbono orgânico em solos tratados com lodo de curtume. Pesquisa Agropecuária Brasileira, v.41, n.7, p.11491155, 2006.

McLauchlan, K. K. Effects of soil texture on soil carbon and nitrogen dynamics after cessation of agriculture. Geoderma, v.136, n.1-2, p.289-299, 2006.

Müller, T.; Hopper, H. Soil organic matter turnover as a function of the soil clay content: consequences for model applications. Soil Biology and Biochemistry, v.36, p.877-888, 2004.

Nelson, D. W.; Sommers, L. E. Total carbon, organic carbon, and organic matter. In: Methods of soil analysis. Part. 2, 2.ed., p.539-579, 1982.

Oades, J. M. The retention of organic matter in soils. Biogeochemistry, v.5, p.35-70, 1988.

Pedroza, J. P.; van Haandel, A. C.; Beltrão, N. E. de M.; Dionísio, J. A.; Duarte, M. E. M. Qualidade tecnológica da pluma do algodoeiro herbáceo cultivado com biossólidos. Revista Brasileira de Engenharia Agrícola e Ambiental, v.10, n.3, p.586-592, 2006.

Rodella, A. A.; Saboya, L. V. Calibration for conductimetric determination of carbon dioxide. Soil Biology and Biochemistry, v.31, p.2059-2060, 1999.

Teles, C. R.; Munaro, C. J.; Cassini, S. T. A.; Collin, H. Modelagem a decomposição aeróbia de lodo de esgoto em solos com diferentes texturas. In: $24^{\circ}$ Congresso Brasileiro de Engenharia Sanitária e Ambiental, 24, 2007, Belo Horizonte. Anais... Belo Horizonte: SBEA, 2007. CD-ROM.

Thomsem, I. K.; Schonning, P.; Olesen, J. E.; Christensen, B. T. $\mathrm{C}$ and $\mathrm{N}$ turnover in structurally intact soils of different texture. Soil Biology and Biochemistry, v.35, p.765-774, 2003.

Vance E. D.; Brookes, P. C.; Jenkinson D. S. An extraction method for measuring soil microbial biomass C. Soil Biology and Biochemistry, v.19, p.703-707, 1987.

Wang, W. J.; Dalal, R. C.; Moody, P. W.; Smith, C. J. Relationships of soil respiration to microbial biomass, substrate availability and clay content. Soil Biology and Biochemistry, v.35, p.273-284, 2003.

Whitmore, A. P. Describing the mineralization of carbon added to soil in crop residues using second-order kinetics. Soil Biology and Biochemistry, v.28, p.1435-1442, 1996. 